\title{
A Unified Framework to Assess Myocardial Function from 4D Images
}

\author{
P. Shi, G. Robinson, A. Chakraborty \\ L. Staib, R. Constable, A. Sinusas, and J. Duncan \\ Departments of Diagnostic Radiology, Electrical Engineering, and Medicine \\ Yale University, New Haven, CT 06520-8042, USA \\ email: xship@noodle.med.yale.edu
}

\begin{abstract}
This paper describes efforts aimed at developing a unified framework to more accurately quantify the local, regional and global function of the left ventricle (LV) of the heart, under both normal and ischemic conditions, using four-dimensional (4D) imaging data over the entire cardiac cycle. The approach incorporates motion information derived from the shape properties of the endocardial and epicardial surfaces of the $\mathrm{LV}$, as well as mid-wall $3 \mathrm{D}$ instantaneous velocity information from phase contrast MR images, and/or mid-wall displacement information from tagged MR images. The integration of the disparate but complementary sources of information overcomes the limitations of previous work which concentrates on motion estimation from a single image-derived source. ${ }^{1}$
\end{abstract}

\section{Introduction}

The measurement of regional myocardial injury due to ischemic heart disease is an important clinical problem. It is the goal of many forms of cardiac imaging and image analysis to measure the regional functions of the left ventricle (LV) in an effort to isolate the location, severity and extent of ischemic or infarcted myocardium. The ability to make these measurements has a variety of benefits, including the idea that serial analysis of regional function is helpful in assessing the efficacy of thrombolytic and other therapeutic agents, and angioplasty [5, 8].

In this paper, we describe a unified framework that integrates the two sets (endocardial and epicardial) of shaped-based estimates of the LV surface motion from our previous work [15], along with estimates of mid-wall motion derived from some of the MR physics-based methods, i.e. phase contrast cardiac MR imaging and MR tagging of myocardium, in an attempt to quantify the $3 \mathrm{D}$ deformation of the LV. The framework is built upon a continuum mechanical model of the left ventricle, and is embedded in a finite element grid. The integration of the disparate but complementary sources of information provides more accurate and robust measurements of the pointwise motion of the entire LV myocardium. We have reviewed much of the relevant literature on endocardial/epicardial wall

\footnotetext{
1 This work was supported by NIH-NHLBI grant R01-HL44803.
} 
motion in our previous work, we will briefly review efforts related to the unified framework we propose here.

MR Tagging of the Myocardium. While the attractiveness of tracking grid lines that can actually be seen in the underlying image has been noted by many investigators [1,21], there are difficulties with the use of MR tagging data for the $4 \mathrm{D}$ analysis of $\mathrm{LV}$ function because: i.) it's difficult to track the tags over the complete LV cycle due to decay of the tag lines and ii.) it's quite difficult to obtain acquisitions and assemble the detected tags into a robust 3D analysis/display. We note, however, the efforts of Young [20] and Prince [7] for assembling MR tagging-derived information into 3D maps of cardiac motion/function.

Phase Contrast Cardiac MR Imaging. Another approach for motion tracking is the use of phase contrast images to decipher local velocity which in turn can be integrated to estimate trajectories of individual points over time [13]. Several investigators have studied the resolution and accuracy of these techniques for tracking myocardial motion and strain [6]. Currently, phase contrast velocity estimates near the endocardial and epicardial boundaries are extremely noisy since the required size of the regions-of-interest (ROI) due to signal-to-noise limitations is so huge that it includes information from outside the myocardial wall. Thus, as with MR tagging, the most accurate LV motion and function information is obtained from the middle of the myocardial wall, and is least accurate near the endocardial and epicardial wall boundaries.

Computer Vision Approaches to Non-Rigid and Cardiac motion. A body of work has emerged from the computer vision community related to the topic of nonrigid motion and the registration of nonrigid surfaces that is directly relevant to the cardiac motion problem. The efforts in nonrigid surface registration include the use of deformable thin-plate splines [3] and octree-spline based volumetric transformation [18], among others. This research attempts to register entire sets of image data and then quantitatively and statistically look for similarities and differences. The more physical-model motivated work of Pentland [9] and Terzopolous [19] is also aimed at solving this embedding problem. In all of these approaches, any estimates of correspondence between individual points on objects are either specifically assumed to be known to aid in solving the problem, or are not considered at all in the solution. The goal of the nonrigid motion recovery is to find point correspondences between two objects over as dense a spatial field as possible. Goldgof [10] has been pursuing surface shape matching ideas, using Gaussian curvature under conformal stretching models. Recently, Metaxas [12] has utilized the mid-wall point correspondences estimated from MR tagging in conjunction with the deformable superquadrics approach. Ayache [11] has been trying to unify boundary shape landmark approach with physically-based framework to segment and track objects simultaneously.

\section{Methods}

We are working towards the development of a unified framework which uses the shape properties of the endocardial and epicardial surfaces, as well as incorporates mid-wall 3D instantaneous velocity information of phase contrast MR images and displacement information of tagged MR images if they are available, 
to track the $4 \mathrm{D}$ trajectories of a dense field of points which sample the myocardial wall over the entire cardiac cycle. Furthermore, it is our intent to derive accurate myocardial motion, LV thickening and strain measures from these trajectories, useful (from both a basic research and clinical standpoint) for the study of the location, severity and extent of ischemic injury.

In this section, we first briefly describe the experimental procedures used for our in vivo MRI-based validation experiments. Next we describe the MR imaging protocols used in this effort. Finally, we describe the basic ideas of our newly developed unified tracking strategy, earlier versions of which (the shapebased tracking of endocardial and epicardial surfaces) have been described more completely in [15]. ${ }^{2}$

\subsection{Experimental Setup for In Vivo MRI-based studies}

Acute Infarct Animal Model. To date, 12 acute experiments yielding 9 usable datasets have been performed on open chested dogs subjected to permanent coronary artery occlusion, resulting in transmural myocardial infarction. A limb lead of the electrocardiogram was continuously monitored, with non-ferromagnetic electrodes. The femoral vein and both femoral arteries were isolated and cannulated for administration of fluids and medications, pressure monitoring, and arterial sampling. The proximal left anterior descending coronary artery was isolated after the first diagonal branch, for placement of an occluder.

Paired endocardial and epicardial markers were placed in 4 locations on the heart. We implanted a small, bullet-shaped copper plug $(1 \mathrm{~mm} \times 2.5 \mathrm{~mm})$ through the myocardium with a hollow metal insertion tube through an 18 gauge needle track. This void marker was loosely tethered to the endocardial surface by an elastic string. The string attached to each endocardial marker passes through the myocardium and is secured to a Gd-DTPA-filled marker sutured onto the epicardial surface. The bright epicardial markers are small, plastic encasings (inner volume $73.81 \mathrm{~mm}^{3}$ ) filled with a solution of saline and Gd-DTPA (200:1). The elastic string attaching the epicardial and endocardial markers keeps the endocardial marker on the endocardial surface during the entire cardiac cycle, without restricting myocardial thickening.

The dogs were positioned in the magnet in the left lateral position for initial 4D imaging under baseline conditions, according to the MR imaging protocols described below. The left anterior descending coronary artery was then occluded, without movement of the animal relative to the imaging planes. $4 \mathrm{D}$ images were acquired again after occlusion. After completion of the acute protocol, hearts were rapidly excised for postmortem imaging and histochemical staining. The hearts were then sectioned in $5 \mathrm{~mm}$-thick slices perpendicular to the long axis of the heart. 3D post mortem injury maps were then reconstructed from digitized photographs of the myocardial slices.

Magnetic Resonance Imaging Protocol. MR imaging was performed on GE Signa 1.5 Tesla scanners with version 4.7 software and hardware using a head

\footnotetext{
${ }^{2}$ We are also testing our approach using high resolution, 4D cine-computed tomographic data, acquired using the Dynamic Spatial Reconstructor (DSR) at the Mayo Clinic in collaboration with Dr. Erik Ritman. The DSR experiments followed roughly the same procedure, but are not described here for brevity.
} 
coil. Axial images through the LV were obtained with the gradient echo cine technique using the following parameters: section thickness $5 \mathrm{~mm}$, no inter-section gap, $42 \mathrm{~cm}$ field of view, TE $14 \mathrm{msec}$, TR $20 \mathrm{msec}$, flip angle 30 degrees, 256x256 matrix and 2 excitations. Images typically were acquired with 2 locations per acquisition and 16 cardiac phases per location. Each slice location acquisition required approximately an 8 minute acquisition time for 2 excitations. This sequence provides images with an in plane resolution of $1.64 \times 1.64 \mathrm{~mm}$ for a $128 \times 128$ matrix and $5 \mathrm{~mm}$ resolution perpendicular to the imaging planes. Temporal resolution is $40 \mathrm{msec}$ for each location acquisition. Example images at 3 slice levels in a single temporal 3D frame are shown in figure 1, with arrows indicating the locations of the implanted validation markers. For 3 of these studies, phase contrast MR images were also acquired in 3 mid-ventricular slices for the same dog heart, which provide mid-wall velocity information for the imaged regions.

\subsection{Image Analysis: A Unified Motion Tracking Framework}

Extended from our previous shape-based motion tracking approach [15], we are developing a unified framework using continuum biomechanical model which utilizes both the LV boundary information (which is available from the shape properties of endocardial and epicardial surfaces) and the mid-wall information (which can be provided by the 3D phase contrast MR images and/or the tagged MR images). ${ }^{3}$ We construct a complete triangular finite element grid which tessellates not only the LV surface sample points, but also the sample points in the mid-wall. The union of all the tetrahedra forms the solid model of the entire left ventricle (see figure 4). The unified motion tracking algorithm then uses this grid to embed the underlying biomechanical constraints of the LV model, and tracks the motion of points everywhere on the entire LV wall using the known displacement information of the sparse endocardial/epicardial surface sample points and/or the mid-wall tagged points, as well as the velocity information of a sparse set of mid-wall points. From the dense motion trajectory field, other LV function measurements such as 3D LV thickening and strain maps, can be obtained. We note the 3D framework is still in development, but we will report some of the initial ideas and 2D results in this subsection.

Generating the Finite Element LV Model. The first step to construct the finite element LV model is to segment the 4D image data on a slice by slice basis to find the LV boundary. This is currently performed by treating the data as if it is consisted of a sequence of temporal 3D frames where there exists a spatial stack of 2D images in each frame, although we are continuing to investigate complete $3 \mathrm{D}$ parametric surface approach as well [17]. We solve the 2D boundary finding problem twice in each slice, once for the epicardial border and once for the endocardial border using a deformable contour approach that we have developed for $2 \mathrm{D}$ boundary finding $[16,4]$. The boundaries found in this plane are now used as a bias and initial estimate for locating the endocardial and epicardial boundaries in the next plane in the stack. This process repeats until all of the contours

\footnotetext{
3 Although the aim of the unified framework is to utilize multiple complementary sources of constraints to achieve more reliable and robust estimates, it will work if only single source of information is available.
} 
that make up the LV surfaces in each frame are completely located. Meanwhile, some mid-wall points of the phase contrast MR images (and landmark mid-wall points of the tagged MR images) are also identified. Finally, the contours that form each endocardial and epicardial surface in each 3D frame as well as the mid-wall sample points identified from phase contrast and tagging images will be stacked. A Delaunay triangulation algorithm is then used to tessellate the stacked LV sample points. A solid finite element LV model which consists of many tetrahedra representing all parts of myocardium is thus generated.

Boundary Displacement Information. The movement of a sparse set of LV surface points is performed by following local surface shape [15]. The tessellated dense set of surface points are used to guide shape calculations, and surface curvature maps over the cardiac cycle for both endocardial and epicardial surfaces are computed through multi-level local surface patch fitting. For each time instant, two sparse subsets of the surface points are created by choosing geometrically significant shape landmark points, one for endocardial surface and the other for epicardial surface. The best matched point for each shape landmark at the next time frame is located using a 3D bending energy model. In this effort, surface patches on the LV are modeled as thin, flexible plates. The strain energy of the deformation required to bend a curved plate or surface patch to a new deformed state is defined as a function of the changes of two principal curvatures, and is invariant to 3D rotation and translation. Under the assumption that each surface patch deforms only slightly and locally within a small time interval, for each sampled point on the first surface, we construct a search area on the second surface. The point within the search window on the second surface that is best matched (i.e. minimizing the bending energy) is chosen as the corresponding point to the one at the first surface, while the bending energy for all the other points inside the window are also recorded to be used as a indicator of the uniqueness of the match. The result of this matching process yields a set of shape-based, best-matched initial motion vectors $\mathbf{D}_{0}(u, v)$ for pairs of surfaces derived from 3D image sequence, as well as information from within each search area as to how confident the match is.

A regularization procedure is then adopted to result in a dense displacement vector field that is an optimal compromise between an adherence term and a smoothness term, with the sparse shape landmark points' initial matches being the only matching constraint to find the dense point correspondence between surfaces. The process is embedded in the irregular triangular surface grid generated from the Delaunay tessellation, and is given by the following expression:

$\mathbf{D}^{*}(\mathrm{u}, \mathrm{v})=\arg \min _{\mathbf{D}} \int_{\mathrm{U}} \int_{\mathrm{V}}\left\{\mathrm{C}_{\mathbf{D}}(\mathrm{u}, \mathrm{v})\left[\mathbf{D}(\mathrm{u}, \mathrm{v})-\mathbf{D}_{\mathbf{0}}(\mathrm{u}, \mathrm{v})\right]^{2}+\left(\frac{\partial \mathbf{D}(\mathrm{u}, \mathrm{v})}{\partial \mathbf{u}}\right)^{2}\right\} \quad \mathrm{d} \mathbf{u}$

In this equation, $[U, V]$ is the domain of the surface at time $t$ in which the grid from the Delaunay triangulation is embedded, $\mathbf{u}=[u, v]^{T}$ is the surface point at time $t, \mathbf{D}^{*}(u, v)$ is the optimal smoothed motion vector field between two surfaces at times $t$ and $t+\delta t, \mathbf{D}_{0}(u, v)$ is the initial motion vector estimate, and $C_{\mathbf{D}}(u, v)$ is the confidence measure matrix which weighs both the goodness and uniqueness of the initial match.

In the new unified framework, the displacement vectors between time frames 
of the shape landmark points will be used only as part of LV deformation constraints, the boundary displacement constraint. In addition, the triangular grid is now a solid volumetric mesh instead of a surface grid.

Mid-Wall Velocity/Displacement Information. The mid-wall region of the 3D phase contrast images can be sampled at a number of points with their instantaneous velocity known at each time frame. This mid-wall information can provide additional constraints to track the LV wall motion. Our group's initial work in this area is documented in [6], in which a stable algorithm that combines forward and reverse integration within a rigorous framework for velocity mapping is presented. Meanwhile, the displacement information from a sparse set of midwall MR tagging landmark points can be computed by following trajectories of some MR tag points over time [14]. This will provide yet another set of constraint to track the motion of the dense field $\mathrm{LV}$ points.

A Unified Framework to Assess Myocardial Function. We have constructed a $3 \overline{\mathrm{D}}$ finite element LV model which has nodes in the mid-wall region, as well as nodes on endocardial and epicardial surfaces. We also have established the displacement vectors for a sparse set of surface shape landmark nodes and midwall tag points, and the velocity information for a sparse set of mid-wall phase contrast nodes. Assuming the boundary and mid-wall nodal points represent the LV reasonably well, a continuum biomechanical LV model based on the theory of finite elasticity and realistic material law is under development. Using the finite element method to solve the deformation of the LV over time, under the known boundary conditions (the displacement and velocity information of some of the nodes) and external forces (i.e. flow pressure, volume change) if available, the equilibrium equations can be established as [2]:

$$
\mathbf{M U}+\mathbf{C} \dot{\mathbf{U}}+\mathbf{K U}=\mathbf{R}
$$

where $\mathbf{M}$ is the mass matrix, $\mathbf{C}$ the damping matrix, $\mathbf{K}$ the stiffness matrix, $\mathbf{R}$ the external load, and $\mathbf{U}$ the nodal displacement vector field. We note that the form in equation (2) describes a very general finite element system, while the known displacement/velocity conditions and the external forces provide more specific constraints for our framework. We want to point out that we intend to use this model to enforce certain real physical constraints related to known cardiac volumes and pressures, as well as realistic biomechanical properties of the myocardium, not just physically analogous ones as described by Pentland [9] and others. Let $\mathbf{U}_{a}$ be the known displacements, $\mathbf{U}_{b}$ the displacements of the nodes with known velocity, and $\mathbf{U}_{c}$ the displacements of the nodes with no prior information, we can rewrite the equilibrium equations in the form ${ }^{4}$ :

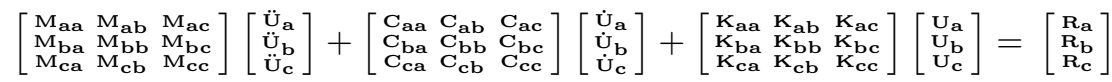

where $\mathbf{U}_{a}$ and $\dot{\mathbf{U}}_{b}$ are prescribed conditions. The iterative solutions of these pre-conditioned equilibrium equations yield displacements for all element nodal points, from which the 3D strain and stress maps can be obtained.

\footnotetext{
${ }^{4}$ For simplicity of the equation, we assume no node has both known displacement and velocity information.
} 


\section{Experiments and Results}

Using the initial form of our unified framework described above, the initial experimentation with the cardiac motion and function analysis is reported here. As mentioned above, to date we have acquired nine usable sets of $4 \mathrm{D}$ MRI data using the acute infarct animal model. The visual results shown here are from two of the MR studies that have been analyzed.

Figure 1 shows three image slices from the same 3D MRI time frame, illustrating the paired markers used for motion tracking validation (white arrows $\Rightarrow$ epicardial markers, black arrows $\Rightarrow$ endocardial markers). Implanted markers' centroids are detected over the entire cardiac cycle, and their trajectories are compared to the algorithm-derived points trajectories in order to validate the accuracy of the developed methodology. Figure 2 illustrates the comparison of algorithm-computed (lighter) trajectories with the marker-derived (darker) trajectories of three endocardial points, moving from end-diastole (ED) to endsystole (ES). The paths are shown relative to the endocardial surface rendered at global end-systole (lowest overall chamber volume). The two most right points have almost identical marker- and algorithm- derived trajectories, while the left one is a little bit off after several time intervals (but they are still very close).

Figure 3 and table 1 are presented to illustrate some of the measurements from which useful physiological parameters will be derived. Figure 3 shows an infarcted endocardial surface roughly in its end-systolic state. On the left, the dense motion trajectories are shown in the normal zone of the infarcted left ventricle, while on the right, the dense trajectories locating in the infarct zone of the same LV are shown. Note in this case, the normal zone has far larger motion than the infarct zone does. Table 1 provides the quantitative comparison of normal and infarct zone measurement (path length) before and after the coronary occlusion surgery which causes the myocardial injury. From the table we observe that for both studies, the normal zone points have relatively stable movement pre- and post- infarction while the infarct zone's mobility drops fifty percent or more.

A key additional measurement of interest to us is that of myocardial thickness and strain. By tracking small, related regions of points on both the LV surfaces (endocardium and epicardium) and mid-wall myocardium over time, we can create transmural and non-transmural measures of myocardial strain and use the radial component of strain to infer thickening changes. Non-transmural strain measures are of great value for predicting early myocardial injury and viability. Figure 4 displays of a tessellated 3D LV wall mesh deforming over time from ED to ES, in the order of left, middle, and right. Figure 5 shows the gray scale maps of three 2D principal strain maps, as well as the principal direction associated with the maximum principal normal strain. Here, we denote large positive strain with light white shade, large negative strain with dark shade, and near zero strain with neutral gray. For any given element, the positive principal strain represents the stretching in the associated direction, the negative one represents the compressing effect.

In addition to reporting the above initial results on the motion/function tracking algorithm, we also report here the results of initial steps taken towards comparing the in vivo algorithm-based measurements to post mortem measures. 


\begin{tabular}{||l||c|c|c|c||}
\hline \hline \multicolumn{1}{||c||}{} & \multicolumn{2}{c|}{ normal zone path length } & \multicolumn{2}{c||}{ infarct zone path length } \\
\hline study & baseline & post - infarction & baseline & post - infarction \\
\hline \hline 1 & $17.07 \pm 2.79 \mathrm{~mm}$ & $14.50 \pm 2.05 \mathrm{~mm}$ & $22.50 \pm 2.13 \mathrm{~mm}$ & $8.76 \pm 2.16 \mathrm{~mm}$ \\
2 & $13.32 \pm 1.67 \mathrm{~mm}$ & $15.79 \pm 2.26 \mathrm{~mm}$ & $14.28 \pm 1.81 \mathrm{~mm}$ & $7.31 \pm 2.35 \mathrm{~mm}$ \\
\hline \hline
\end{tabular}

Table 1. Table of path lengths of endocardial normal and infarct zones of two canine MR studies, under baseline and post-infarction conditions. These data were average path lengths within each zone (defined by points' closeness to the implanted markers). Note the relative stableness of the normal zone path lengths before and after infarction, and the big drop of the infarct zone path lengths after coronary occlusion.

Ultimately, the image frame in each of the pre- and post- infarction studies whose markers best match the post mortem markers by minimizing the overall Euclidean distance between the sets of points will be chosen as the in vivo myocardial injury reference frame for that study. Next, color masks representing the infarct zone and normal zone that were found on the post mortem surface are mapped onto both the pre- and post- infarction in vivo endocardial reference surfaces. Classification of myocardium from in vivo image-derived measurement will be compared to the post mortem measures. Results from the first step of this process, showing two views of the computer-reconstructed, color coded 3D post mortem surface, are shown in figure 6 . Note that the right figure shows the non-transmural nature of the myocardial injury in this study.

\section{Summary}

We have described our ongoing efforts aimed at developing a unified framework to more accurately quantify the local, regional and global function of the left ventricle $(\mathrm{LV})$ of the heart, under both normal and ischemic conditions. Our approach incorporates multiple sources of constraints, including motion information derived from the shape properties of the endocardial and epicardial surfaces, mid-wall 3D instantaneous velocity information from phase contrast MR images, and/or mid-wall displacement information from tagged MR images.

Ongoing and future work includes developing more sophisticated continuum biomechanical model of the myocardium based on viscoelastic material law, incorporating temporal periodic characteristics of the heart motion into the current framework.

\section{References}

1. L. Axel and L. Dougherty. MR imaging of motion with spatial modulation of magnetization. Radiology, 171:841-845, 1989.

2. K. Bathe and E. Wilson. Numerical Methods in Finite Element Analysis. PrenticeHall, New Jersey, 1976.

3. F. L. Bookstein. Principal warps: Thin-plate splines and the decomposition of deformations. IEEE Trans. on Patt. Anal. and Mach. Intell., pages 567-585, 1989. 
4. A. Chakraborty, L. H. Staib, and J. S. Duncan. An integrated approach to boundary finding in medical images. In Proceedings of the IEEE Workshop on Biomedical Image Analysis, pages 13-22, 1994.

5. J. Chesebro and G. Knatterrud et al. Thrombolysis in myocardial infarction (TIMI) trial phase I: A comparison between intravenous tissue plasminogen activator and intravenous streptokinase. Circulation, 76:142-154, 1987.

6. R.T. Constable, K. Rath, A. Sinusas, and J. Gore. Development and evaluation of tracking algorithms for cardiac wall motion analysis using phase velocity MR imaging. Magnetic Resonance in Medicine, 32:33-42, 1994.

7. T. S. Denney and J. L. Prince. 3d displacement field reconstruction from planar tagged cardiac MR images. In Proceedings of the IEEE Workshop on Biomedical Image Analysis, pages 51-60, 1994.

8. W. Grossman. Assessment of regional myocardial function. JACC, 7(2):327-328, 1986.

9. B. Horowitz and S. Pentland. Recovery of non- rigid motion and structure. In IEEE Conf. on CVPR, pages 325-330, Maui, June 1991.

10. C. Kambhamettu and D. Goldgof. Point correspondence recovery in non-rigid motion. In IEEE Conf. on CVPR, pages 222-227, June 1992.

11. C. Nastar and N. Ayache. Non-rigid motion analysis in medical images: a physically based approach. In Information Processing in Medical Imaging. SpringerVerlag, 1993.

12. J. Park, D. Metaxas, and A. Young. Deformable models with parameter functions: Application to heart wall modeling. In IEEE Conf. on CVPR, pages 437-442, June 1994.

13. N. J. Pelc, A. Shimakawa, and G. H. Glover. Phase contrast cine MRI. In Proceedings of the 8th Annual SMRM, page 101, Amsterdam, 1989.

14. P. Shi, A. Amini, R. T. Constable, and J. Duncan. Tracking tagged MR images with energy-minimizing deformable grids. In Proceedings of the 18th IEEE Annual Northeast Bioengineering Conference, pages 133-134, 1992.

15. P. Shi, A. Amini, G. Robinson, A. Sinusas, R. T. Constable, and J. Duncan. Shape-based $4 \mathrm{~d}$ left ventricular myocardial function analysis. In Proceedings of the IEEE Workshop on Biomedical Image Analysis, pages 88-97, 1994.

16. L. H. Staib and J. S. Duncan. Parametrically deformable contour models. IEEE Trans. on Patt. Anal. and Mach. Intell., 14(11):1061-1075, 1992.

17. L. H. Staib and J. S. Duncan. Deformable fourier models for surface finding in 3d images. In Visualization in Biomedical Computing, pages 90-104, Oct. 1992. SPIE 1808.

18. R. Szeliski and S. Lavallee. Matching 3 d anatomical surfaces with non-rigid deformations using octree-splines. In Proceedings of the IEEE Workshop on Biomedical Image Analysis, pages 144-153, 1994.

19. D. Terzopolous and D. Metaxas. Dynamic 3d models with local and global deformation: Deformable superquadrics. IEEE Trans. on Patt. Anal. and Mach. Intell., 13(17), 1991.

20. A. A. Young, D. L. Kraitchman, and L. Axel. Deformable models for tagged MR images: Reconstruction of two- and three-dimensional heart wall motion. In Proceedings of the IEEE Workshop on Biomedical Image Analysis, pages 317-323, 1994.

21. E. Zerhouni and et. al. Tagging of the human heart by multiplanar selective RF saturation for the analysis of myocardial contraction. In Abstracts of the Ann. Meeting of the Soc. of MR in Imaging, page 10, San Francisco, 1988.

This article was processed using the $\mathrm{IAT}_{\mathrm{E}} \mathrm{X}$ macro package with LLNCS style 


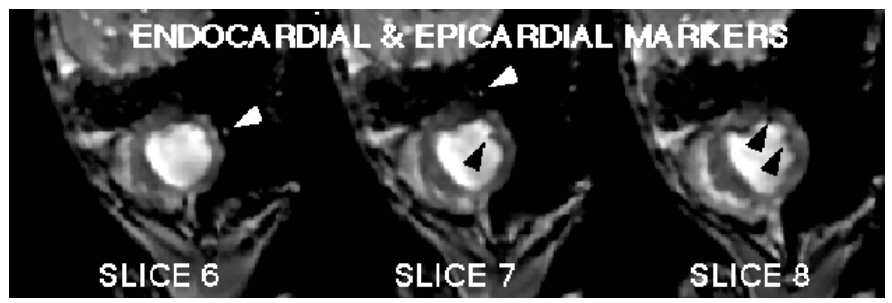

Fig. 1. Three slices from the same 3D MRI time frame, illustrating the paired markers used for motion tracking validation (white arrows $\Rightarrow$ epicardial markers, black arrow $\Rightarrow$ endocardial markers).

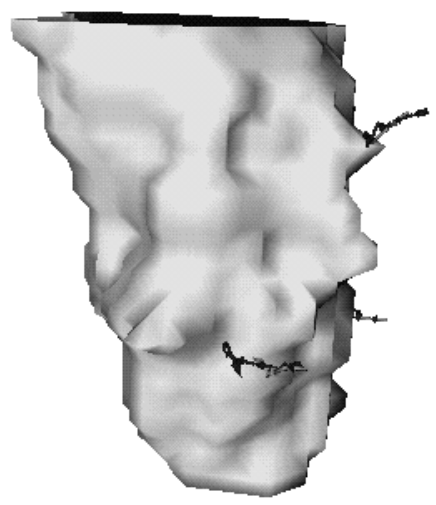

Fig. 2. Comparison of algorithm-computed (lighter) and marker-derived (darker) trajectories of three endocardial points, moving from ED to ES. The paths are shown relative to the endocardial surface rendered at global end-systole. The two right most points have almost overlapping marker- and algorithm- derived trajectories, while the left one is a little bit off after several time intervals.
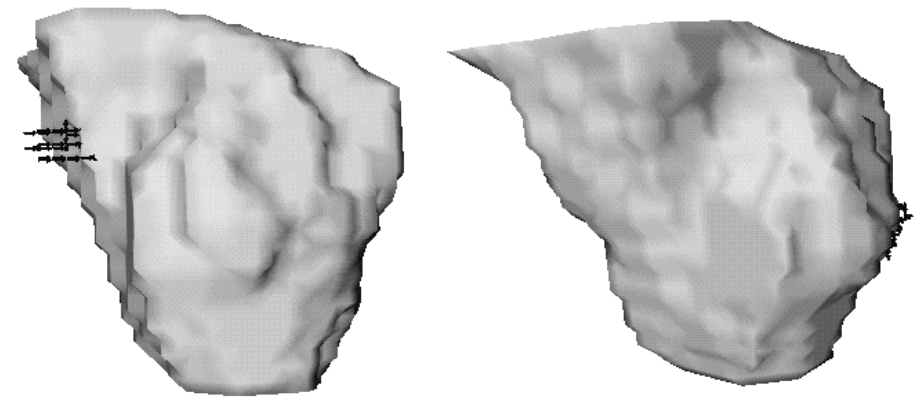

Fig. 3. Display of two sets of dense motion trajectories in the normal and infarct zones of the endocardial surface of an infarcted left ventricle, relative to the surface rendered at global end-systole. The normal zone is shown on the left, the infarct zone is on the right. Note the relatively smaller motion in the infarct zone. (See table 1 for quantitative details). 

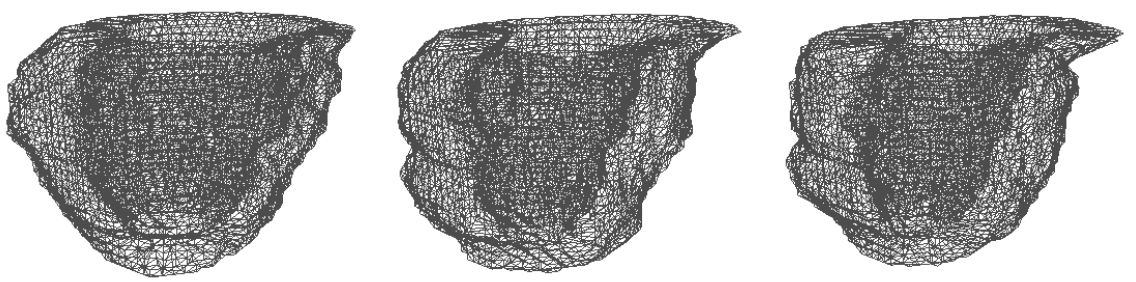

Fig. 4. 3D displays of a tessellated LV wall deforming from ED to ES. Left: end-diastole; Middle: middle of the contraction; Right: end-systole.
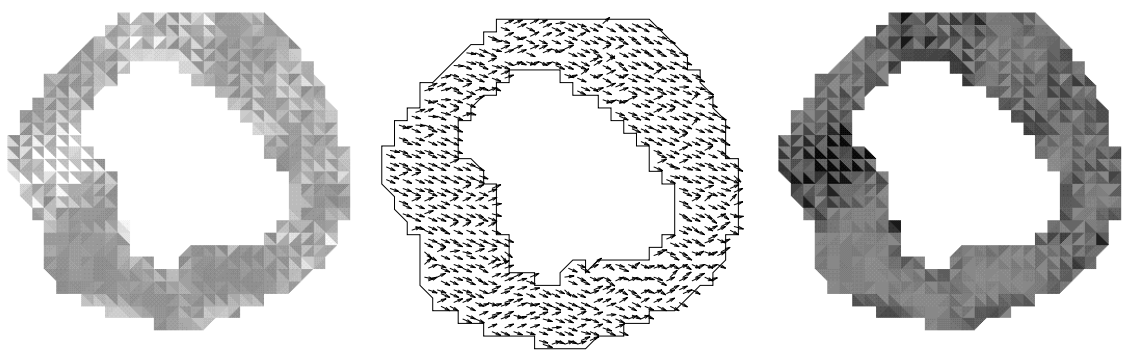

Fig. 5. 2D principal strain maps at ED: maximum principal strain, direction associated with maximum principal strain, and minimum principal strain
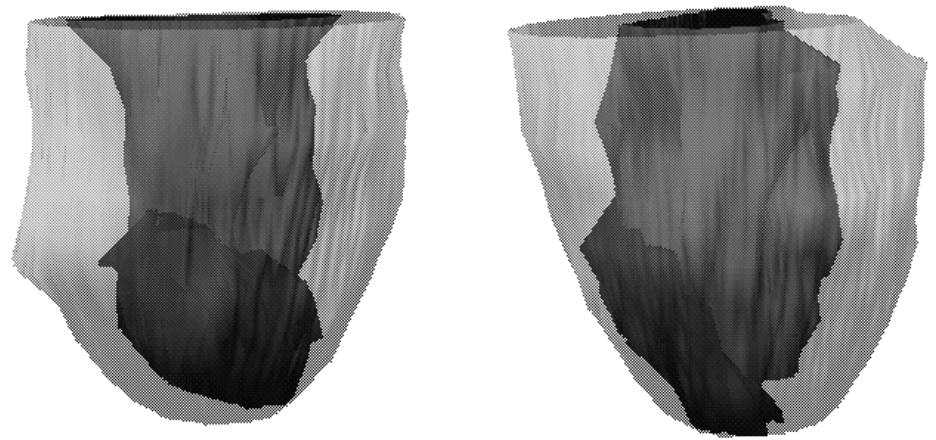

Fig. 6. 3D displays of a reconstructed post mortem LV wall from two views: endocardium (inner lighter opaque), epicardium (outer transparent), and injury region (lower darker opaque). Note the right figure shows the non-transmural nature of the myocardial injury. 